
internationales

vol. 30 - $n^{\circ} 2$ | 2014

Composer (avec) la frontière. Passages, parcours migratoires et échanges sociaux

\title{
Négocier sa place chez l'autre. Les migrants dans les espaces palestiniens à Beyrouth
}

Negotiating a Place. Migrant Workers settle in Beirut's Palestinian Spaces

Negociar su lugar en territorio ajeno. Inmigrantes en espacios palestinos de

Beirut

\section{Assaf Dahdah}

\section{CpenEdition}

Journals

Édition électronique

URL : https://journals.openedition.org/remi/6859

DOI : $10.4000 /$ remi.6859

ISSN : $1777-5418$

Éditeur

Université de Poitiers

Édition imprimée

Date de publication : 1 juin 2014

Pagination : 25-47

ISBN : $979-10-90426-22-1$

ISSN : 0765-0752

Référence électronique

Assaf Dahdah, « Négocier sa place chez l'autre. Les migrants dans les espaces palestiniens à

Beyrouth », Revue européenne des migrations internationales [En ligne], vol. 30 - n² | 2014, mis en ligne le 01 juin 2017, consulté le 16 avril 2022. URL : http://journals.openedition.org/remi/6859 ; DOI : https://doi.org/10.4000/remi.6859 


\section{Négocier sa place chez l'autre. Les migrants dans les espaces palestiniens à Beyrouth}

\section{Assaf Dahdah ${ }^{1}$}

Aborder la géographie palestinienne de Beyrouth signifie généralement l'étude des camps et de leurs populations caractérisées par l'exil (Doraï, 2006) : une histoire qui oscille entre l'altérité fraternelle, les conflits et le " présent sans fin " de l'exode (Agier, 2012). Espaces de relégation, les camps palestiniens se sont pourtant progressivement transformés en quartiers beyrouthins des marges de la ville, certes partiellement intégrés dans le tissu urbain qui compose la première couronne de la capitale libanaise. De cette situation ambivalente entre camp et quartier naît une tension : d'un côté une identité palestinienne encore profondément animée par l'exode et la résistance (Al Husseini, 2012), dont les camps restent des "géosymboles " (Bonnemaison, 2000) ; de l'autre, un processus de dilution de ces enclaves dans une ville dont ils ne semblent plus être que des quartiers parmi d'autres, même s'ils restent perçus comme les " espaces des autres" (Agier, $2013: 72)$. Cette transition entre camp et ville est révélée sinon accentuée par l'installation de travailleurs migrants africains et asiatiques. Ils sont assurément des acteurs de leur transformation, contribuant ainsi à les intégrer davantage aux dynamiques métropolitaines. Refuges et espaces d'opportunité, les périphéries pauvres de Beyrouth et dans une moindre mesure les camps palestiniens sont devenus des lieux de sociabilité et $\mathrm{d}^{\prime}$ activités économiques, et enfin des portes d'entrée dans la ville². De nombreux migrants, dont ceux qui ne possèdent ni autorisation de séjour (ikami) ni de travail (ijazet 'amal), résident dans ces marges où les autorités libanaises ne pénètrent qu'exceptionnellement.

À bien des égards assimilés à des territoires de non-droit pour des apatrides (Hanafi et Long, 2012), les camps palestiniens constituent dorénavant des lieux $d^{\prime}$ installation pour des migrants sans droits ${ }^{3}$. Cependant, la rencontre entre

\footnotetext{
1 Doctorant et ATER en géographie, Aix-Marseille Université/CNRS, Telemme (UMR 7303), 5 rue du Château de I'Horloge, BP 647, 13094 Aix-en-Provence cedex 2 ; assaf.dahdah@ univ-amu.fr

2 Les loyers dans les quartiers centraux atteignent des coûts élevés pour les citadins aux revenus modestes. Les Libanais comme les étrangers parviennent alors à se loger dans ces secteurs défavorisés (Fawaz, 2007).

3 Seuls quelques travaux font état de la présence de travailleurs non arabes spécifiquement dans les camps palestiniens (Doraï, 2010) ou plus généralement dans les quartiers paupérisés de la capitale libanaise (Deboulet et Hily, 2009 ; Deboulet, 2012).
} 
le monde de l'exode et celui de la migration de travail ne s'effectue pas sans difficultés. Ainsi le constat d'une mixité voire d'un certain cosmopolitisme dans les marges ne va pas, bien souvent, sans celui d'un contrôle social et spatial strict. Une certaine violence ponctue parfois les modalités d'échanges entre " anciens invités " et " nouveaux invités ", comme les désignent des Palestiniens interrogés. Conséquences des réalités politiques et économiques qui voient les camps palestiniens devenir des quartiers paupérisés (De Souza Araujo Dias, 2009 ; Hanafi, 2006), les enjeux liés à la présence des migrants doivent être mis en perspective avec les stratégies de certains résidents et propriétaires qui tentent avant tout de se constituer de nouvelles sources de rentes en profitant de la présence et de la vulnérabilité des travailleurs étrangers. Et si les migrants y trouvent une place, celle-ci reste largement caractérisée par l'incertitude et I'instabilité. En effet, leur " droit à l'espace " (right to space) (Soja, 2010) s'avère une négociation permanente : rares sont ceux qui parviennent à acquérir une légitimité tandis que la majorité peut être expulsée à tous moments par les résidents des camps et "les commerçants installés ". En somme, la mixité inhérente à la présence des travailleurs migrants et l'extériorité de ces acteurs vulnérables aux enjeux politiques locaux et régionaux interrogent avec acuité le fonctionnement et l'identité même des camps et des groupements ${ }^{4}$. L'objectif de cet article est donc de rendre compte de ces processus socio-spatiaux en analysant la place et le statut de ces " nouveaux étrangers " dans les espaces palestiniens de Beyrouth.

Dans un premier temps, je décrirai brièvement la situation des migrants au Liban et les trajectoires susceptibles de les conduire dans certains des secteurs relégués de la capitale dont je dresserai ensuite un état des lieux succinct. Dans un deuxième temps, j'aborderai les modalités d'installation des travailleurs africains et asiatiques parmi les populations palestiniennes et arabes à travers la question de l'accès au logement et des stratégies des propriétaires dans le camp de Mar Elias. Enfin, à partir des exemples du marché de Sabra et du groupement de Da'ouk, je rendrai compte des stratégies développées par des migrants dans ces espaces extrêmement hiérarchisés ${ }^{5}$.

\section{De la migration contractualisée au camp palestinien}

Afin de saisir les modalités d'installation des travailleurs migrants dans les espaces palestiniens, il convient de rendre compte d'une part des conditions de vie et de travail des ressortissants africains et asiatiques non arabes au Liban imposées par le cadre normatif de la kafala, et d'autre part de la place des étrangers sur le marché du travail libanais ainsi que des représentations sociales qui en découlent. Une courte présentation des secteurs palestiniens étudiés permettra de dresser un tableau de leurs réalités géographiques; entre reléga-

\footnotetext{
4 Un camp palestinien ou mukhayyam est officiellement reconnu par les autorités libanaises et géré par I'Unrwa (United Nations Relief and Works Agency for the Palestine Refugees in the Near East). Un groupement ou tajamou' constitue une extension d'un camp. Occupation illégale de terrains publics ou privés, un groupement n'est pas reconnu par les autorités libanaises, mais il est géré de facto par les habitants et les organisations palestiniennes (Doraï, 2006).

5 Ce travail s'appuie sur une centaine d'entretiens semi-directifs et des observations de terrain menés dans le cadre de ma thèse entre 2011 et 2014.
} 


\section{Carte 1 : Camps et groupement palestiniens au sud de Beyrouth en 2014}

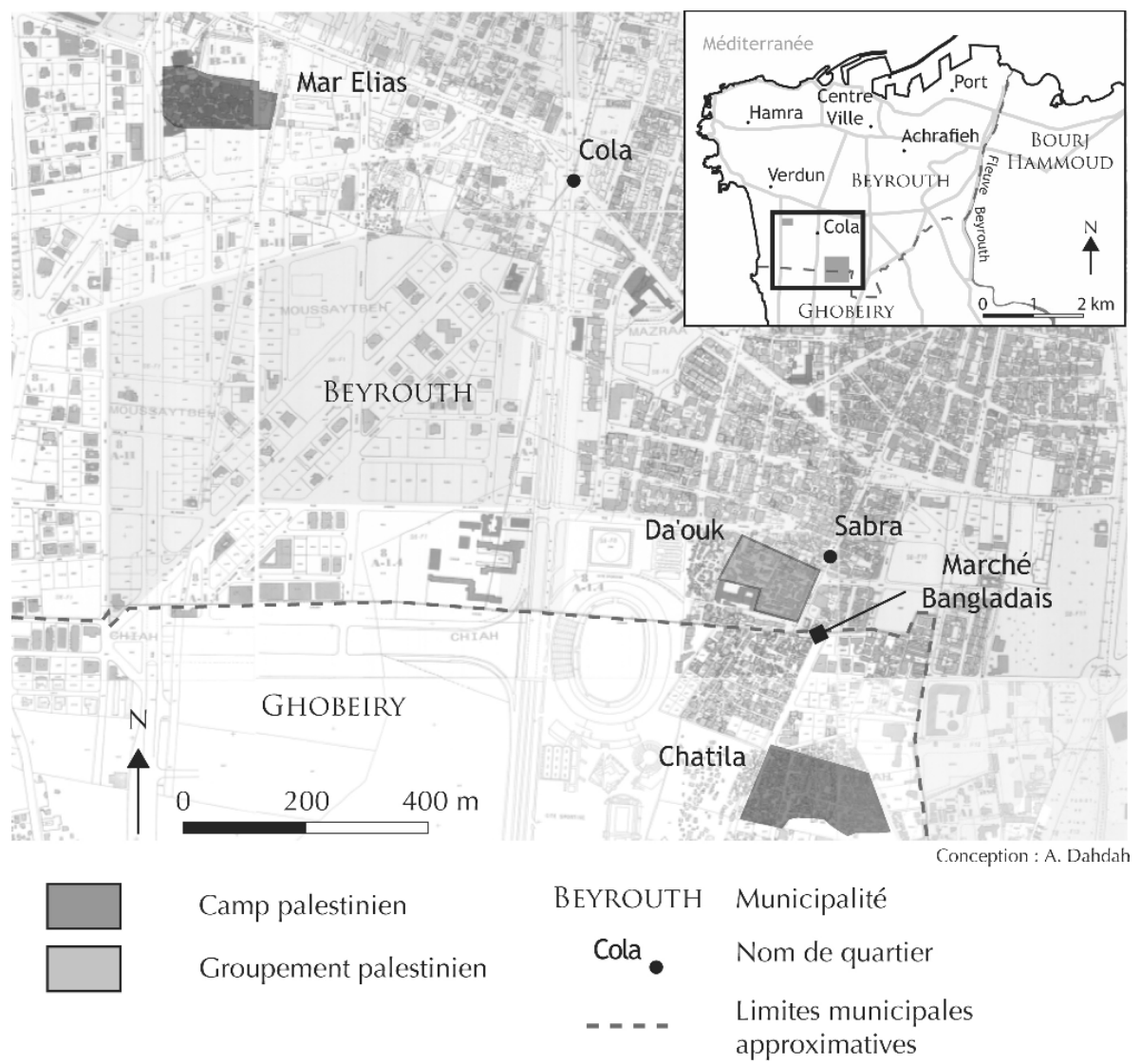

Sources : Cadastre de Beyrouth (2004) (fond de carte) ; enquête personnelle de terrain (2011-2014). 
tion et paupérisation, exil et urbanité, camps et regroupements s'inscrivent dans un temporaire qui s'éternise et les nécessités du quotidien dans lesquelles vient s'immiscer une nouvelle altérité.

\section{Les travailleurs migrants sur le marché du travail libanais}

Depuis la fin des années 1970, le Liban s'inscrit dans le marché international du travail et importe une main-d'œuvre non arabe bon marché dans un contexte national et régional de guerres, de replis communautaires et de développement des économies pétrolières du Golfe (Jureidini, 2003). Parallèlement, les crises économiques et financières déstabilisent profondément les pays africains et asiatiques dont certains comme les Philippines et le Sri Lanka développent des politiques d'exportation de leur main-d'œuvre (Cox, 2006) par le biais d'institutions gouvernementales spécialisées (Dahdah, 2014). Dans le secteur de la domesticité notamment et à l'instar des pays du Golfe, le Liban, malgré les guerres (1975-1990), organise la venue de travailleurs asiatiques et africains qui vont progressivement remplacer les employées arabes - libanaises, syriennes et palestiniennes - (Jureidini, 2003). On assiste à une " désarabisation " du marché du travail pour reprendre le terme qu'emploie Geisser à propos d'Israël (2012). Mais à la différence de la main-d'œuvre syrienne historiquement présente au Liban (Chalcraft, 2009), la migration en provenance d'Asie et d'Afrique reste régulée par la pratique de la kafala qui place tout travailleur étranger sous la tutelle d'un Libanais (le kafil). Les femmes doivent être logées par leurs employeurs sans qu'aucune forme de protection légale ne soit effectivement appliquée puisque les domestiques ne sont pas couvertes par le droit du travail (Longuenesse, 2013). Elles subissent ainsi une violence à la fois structurelle (Gardner, 2010) et symbolique (Moukarbel, 2009). Dans ce contexte, le marché de la main-d'œuvre africaine et asiatique au Liban, qui s'appuie sur un réseau international d'agences de recrutement, $\mathrm{n}^{\prime}$ a cessé de se développer ${ }^{6}$. On estime actuellement le nombre de travailleurs non arabes au Liban à plus de 200000 personnes $^{7}$, soit environ $15 \%$ de la population active libanaise ${ }^{8}$.

Organisé pour limiter l'installation des travailleurs étrangers sur le territoire libanais et empêcher toute possibilité de mobilité sociale et spatiale grâce à un partenariat entre les autorités publiques, les agences privées et les employeurs, le système de la kafala est dans les faits contourné par les migrants, mais aussi par les employeurs eux-mêmes (Bret, 2011). Un nombre croissant de migrants s'installent au Liban et travaillent en dehors des secteurs où les autorités

6 Selon I'ancien ministre du Travail Charbel Nahas interrogé en mars 2012, on estime qu'environ 600 agences de recrutement travaillent légalement au Liban.

7 Lire à ce propos I'article de Jureidini (2012). Selon des responsables associatifs libanais, le nombre de travailleurs non arabes serait a priori plus proche de 400000 personnes.

8 La population libanaise est évaluée à environ 4 millions de résidents dont près de 1,5 million d'actifs, auxquels s'ajoutent la main-d'œuvre syrienne estimée - 300000 personnes - (Chalcraft, 2009), les réfugiés palestiniens enregistrés - plus de 450000 personnes - (UNRWA, 2014) et syriens enregistrés - plus de 1 million de personnes (UNHCR, 2014). Du fait de la guerre en Syrie et de I'afflux croissant et permanent de réfugiés, leur nombre reste approximatif et la distinction entre cette catégorie et celle qualifiée de " main-d'œuvre " est inévitablement floue. Enfin, les chiffres avancés ici restent dans l'ensemble très imprécis, car les données officielles libanaises sont généralement peu fiables et toujours inférieures à la réalité quand il s'agit d'informations ayant trait à l'immigration de travail et politique. 
souhaitent les confiner. Du fait de la marge de manœuvre restreinte imposée par la kafala, et des pressions morales et physiques exercées par nombre d'employeurs sur leurs employés, une part non négligeable de travailleurs embauchés légalement fuit son lieu de travail - principalement les domestiques. De plus, ils sont également susceptibles de se retrouver sans kafil, conséquence du désengagement de ce dernier après un désaccord sur le niveau des revenus et les rythmes imposés. Enfin, nombreux sont ceux qui résident et travaillent également au Liban grâce à un système dit de ghost sponsorship, autrement dit avec une tutelle organisée à l'amiable et généralement contre rétribution entre un Libanais et un travailleur étranger ${ }^{9}$. À ces situations administratives multiples et parfois confuses viennent s'ajouter les faibles revenus des travailleurs étrangers. Si les Libanais ne bénéficient certes pas tous du revenu minimum légal - évalué à environ 500 dollars US par mois -, cette réalité est courante pour les travailleurs étrangers - arabes et non arabes - dont le niveau de rémunération et les conditions de travail restent largement à la discrétion de l'employeur. Une situation généralisée dans le secteur de la domesticité où, conséquence de l'absence de protection légale, l'exploitation des employées conduit à des drames récurrents ${ }^{10}$; moins médiatisés et relevant pourtant du droit du travail, les conflits sociaux liés aux mauvaises conditions d'embauche des étrangers dans les sociétés d'entretien ou dans le secteur industriel sont également fréquents (Wehbe, 2012). L'instabilité administrative, sociale et économique influence largement les trajectoires des migrants dans la ville et tout particulièrement celles des femmes. En situation de clandestinité et/ou de précarité, les migrants s'orientent alors vers les périphéries où les autorités libanaises sont absentes, les propriétaires peu regardants sur le statut administratif des locataires et où les loyers restent abordables.

\section{Chercher refuge dans les marges urbaines}

L'histoire contemporaine de Beyrouth est en grande partie liée à celle de ses marges qui ont accueilli au cours du XXle siècle les populations libanaise, syrienne, kurde, palestinienne et arménienne déplacées ou quittant les campagnes; et leur installation autour de la capitale a constitué ce que certains ont appelé la " ceinture de misère " (hizam el-bû's) (Bourgey et Pharès, 1973). Une dénomination certes politique qui distingue la banlieue de la ville " civilisée " alors assiégée (Harb, 2003), mais qui a le mérite de mettre en lumière des situations de grande pauvreté dans une métropole marquée par de fortes inégalités.

Les populations arménienne et palestinienne se sont installées à la suite de conflits, de massacres et d'expulsions - 1915-1920 et 1939 pour les Arméniens et 1948-1967 pour les Palestiniens -, notamment dans des camps autour de la capitale libanaise $^{11}$-Tiro, Karantina, Sandjak, Dbayeh à l'est, Chatila, Mar Elias et

9 Selon des migrants interrogés, les sommes peuvent avoisiner plusieurs centaines de dollars US et il n'est pas garanti que le potentiel kafil respecte son engagement une fois rémunéré.

10 Lire à cet égard le rapport de I'organisation Human Rights Watch (2010) et Frantz (2014).

11 Certains camps au Liban comme celui de Rashiddiyeh à côté de Tyr ou Karantina à Beyrouth, seront successivement occupés par les Arméniens et les Palestiniens (Doraï, 2010). 
Bourj al-Brajneh au sud ${ }^{12}$. Tandis que l'exode arménien évolue vers une intégration dans le tissu national libanais et urbain beyrouthin avec l'appui des autorités françaises mandataires (Tachjian, 2009), les réfugiés palestiniens vivent toujours au Liban comme une population apatride, stigmatisée et sans droits subissant les crises économiques et politiques récurrentes ${ }^{13}$ (Al Husseini et Doraï, 2013). Dans ce contexte et dans l'attente d'un hypothétique retour, le temps long de l'exil transforme les camps en ghettos urbains selon la définition qu'en propose Agier (2013: 31-33) : " [ces] espaces à part, séparés, lieux précaires de relégation et au devenir incertain [...] [animés par ces] processus inédits de formation urbaine qui prennent racine dans des camps, des campements informels et toutes sortes de hors-lieux ayant fonction de refuge ".

L'aire de Sabra-Chatila ${ }^{14}$ constitue actuellement un continuum spatial que I'on pourrait qualifier de " micro-fragmenté " (Navez-Bouchanine, $2001: 110$ ) en une mosaïque de territoires densément peuplés et paupérisés sous le contrôle des différentes factions palestiniennes et libanaises. Le marché populaire de Sabra attire des citadins de toute la ville à la recherche de produits bon marché viande, fruits et légumes, tissus, vêtements, musique et matériel électronique notamment - et autres marchandises et services illicites - drogues, prostitution. Depuis 2011, cette place marchande se transforme avec l'arrivée croissante de nouveaux commerçants, des Bangladais qui prennent place tous les dimanches au niveau du carrefour où sont regroupés les bouchers, à la limite entre les Municipalités de Beyrouth et de Ghobeiry. Par ailleurs, une population bangladaise, éthiopienne et sri lankaise importante trouve également à se loger dans les quartiers, camps et groupements alentour.

Le camp de Mar Elias se situe dans les limites municipales de Beyrouth. II est le plus petit camp palestinien du Moyen-Orient puisqu'il compte officiellement environ 650 réfugiés palestiniens enregistrés auprès de la United Nations Relief and Works Agency for Palestine Refugees in the Near-East (UNRWA, 2014) ; officieusement plus de 1500 Palestiniens et Libanais y résident. Bâti en 1952 sur des terres mises à disposition par l'évêché rom (grec) orthodoxe pour accueillir des Palestiniens chrétiens de Galilée (Sfeir, 2008), Mar Elias ressemble aujourd'hui, contrairement à Sabra et Chatila, à une petite enclave apaisée dans un quartier en cours d'urbanisation (Doraï, 2010). En plus des résidents palestiniens et libanais, le président du Comité populaire (lejneh cha'biyeh) de Mar Elias (interrogé en mai 2012) estime à plus de 500 le nombre d'étrangers

12 On compte officiellement douze camps palestiniens au Liban, trois ayant été détruits pendant les guerres libanaises (1975-1990) et jamais reconstruits.

13 Les Palestiniens n'ont officiellement pas accès à la nationalité libanaise, ni à la propriété privée - foncière et immobilière - ni à la plupart des secteurs du marché du travail quand bien même les autorités soutiennent la cause palestinienne et leur droit au retour. Cette politique de non intégration est donc censée favoriser et soutenir le retour en Palestine en rendant I'installation impossible (tawtin); les rapports de force confessionnels au Liban expliquent dans une large mesure cette politique à l'égard des Palestiniens.

14 Les camps et les regroupements palestiniens situés à la limite sud de Beyrouth ont été rendus tristement célèbres suite aux massacres et aux exactions perpétrés à l'encontre des réfugiés durant les guerres libanaises, notamment à Sabra et Chatila au cours des années 1980 : massacres menés successivement par les forces des milices chrétiennes appuyées par I'armée israélienne en 1982, mais également par l'armée syrienne et la milice chiite Amal entre 1985 et 1987 (Sayigh, 1994). 
résidant dans l'enceinte du camp, dont près de 300 migrants non arabes, principalement des Éthiopiennes, des Soudanais et des Bangladais ${ }^{15}$, les autres étant des familles de réfugiés et des travailleurs syriens. Au total, on évalue à plus de 2000 les personnes résidant à Mar Elias. On observe que la présence des migrants non arabes a conduit à un développement du commerce dans l'enceinte du camp avec deux salons de coiffure éthiopiens ouverts en 2012 et la création par des commerçants palestiniens de services spécifiques à destination des migrants - notamment la vente de pain éthiopien endjara et d'épices pour les Bangladais. Cependant ces initiatives restent limitées dans un contexte marqué par l'informalité, la pauvreté, et le racisme, que ce soit dans le quartier et sur le marché de Sabra, ou dans le camp de Mar Elias.

\section{Trajectoires résidentielles et marché locatif à Mar Elias}

Le premier argument généralement avancé par les migrants interrogés afin de justifier leur présence dans les périphéries pauvres de Beyrouth est le prix abordable des locations. Les camps palestiniens ne dérogent pas à cette règle et Mar Elias est à cet égard exemplaire. Les migrants disent faire ce choix en connaissance de cause et certains refuseraient même de vivre ailleurs. Mais nombreux s'y sentent également à l'étroit et sous contrôle. Cet encadrement n'est pas occulté par les propriétaires palestiniens et libanais qui tiennent à se prémunir contre toute atteinte à leur réputation dans le camp et par extension à celle du camp lui-même. La présence des étrangers, et plus particulièrement celle des femmes célibataires, comporterait selon eux un risque et leur surveillance serait alors indispensable et légitime. Nonobstant cette crainte, le marché de la location aux migrants constitue une opportunité de rente non négligeable et certains propriétaires n'hésitent pas à construire et à aménager des logements qui leur sont entièrement dédiés.

\section{Contraintes et opportunités résidentielles dans un camp}

Organisé autour de petites ruelles propres, le camp de Mar Elias ne connaît pas les maux de Chatila et de Bourj al-Brajneh - insalubrité, exiguïté, insécurité. Comme le soulignent les autorités palestiniennes locales, cette petite enclave palestinienne de Beyrouth se trouve à proximité de bâtiments officiels - ministère de I'Éducation nationale, siège de I'Unesco et d'une caserne militaire. II s'agit de ne pas attirer l'attention des autorités libanaises afin de ne pas remettre en question la situation géographique du camp, dans les limites municipales de la capitale - où l'électricité n'est en principe rationnée que trois heures par jour -, à proximité des centralités économiques et universitaires de la ville - les quartiers de Verdun et de Hamra notamment à l'ouest de Beyrouth -, et des transports en

15 Les ressortissants des Philippines, d'Éthiopie et du Sri Lanka, principalement venus dans le cadre de la domesticité, sont presque exclusivement des femmes. Une situation différente pour les Bangladais qui présentent un sex-ratio plus équilibré. Selon des données obtenues auprès du ministère du Travail, les migrants non arabes sont à $90 \%$ des femmes. 
commun de Cola ${ }^{16}$. C'est d'ailleurs dans ces quartiers qu'une part importante des migrants interrogés travaille.

Lorsque I'on pénètre dans Mar Elias, il n'est pas fréquent de croiser les migrants qui y résident. Respectant la tranquillité du camp et les recommandations des propriétaires, leur discrétion est assez remarquable. Selon le président du Comité populaire, les migrants sont présents à Mar Elias depuis le début des années 2000, même si des Soudanais se seraient installés dans le camp dès les années 1970. En colocation, seuls ou en famille, ils louent des chambres ou des appartements. Les logements visités sont généralement sombres et dans un état vétuste du fait de I'humidité et de la piètre qualité du bâti. Cependant, certains migrants occupent également des logements dans des immeubles nouvellement construits.

Lors des enquêtes réalisées en 2012, les loyers oscillaient alors entre 130 dollars US (95 euros) pour une chambre sans sanitaire ni cuisine, et 400 dollars US (295 euros) pour un appartement comprenant trois pièces dont une transformée en salon de coiffure éthiopien. Si les personnes interrogées se disent généralement satisfaites de leur logement, l'augmentation continue du coût des loyers et l'état des logements restent malgré tout susceptibles de remettre en cause la présence de certaines dans le camp. En effet, une demande croissante de la part de réfugiés - syriens et palestiniens de Syrie nouvellement arrivés au Liban - provoque une inflation des loyers et les propriétaires se montrent plus exigeants. L'exemple de M. et T., un couple soudanais-éthiopien installé à Mar Elias depuis 2010, illustre la difficulté de conserver et de trouver un logement décent à un prix correct.

M., Soudanais de la région de Kurdufan, est entré illégalement au Liban depuis la Syrie en 2000. Ayant obtenu des papiers de séjour et de travail, il est maintenant employé comme chauffeur par l'ambassade du Qatar. Arrivée au Liban en 2006 avec un contrat d'employée domestique, T. travaille comme femme de ménage free-lance. Le couple vit relativement confortablement, d'autant plus que M. a développé une petite entreprise qui filme les événements - mariages et fêtes religieuses - des migrants africains au Liban. Résidant dans la banlieue Sud (Dahiyeh) jusqu'en 2010, les problèmes sécuritaires et les contrôles récurrents effectués par le Hezbollah ont incité le couple à quitter la région pour s'installer à Mar Elias, où selon $\mathrm{M}$. le lieu est plus calme. T. et $\mathrm{M}$. ont loué jusqu'en juin 2012 une chambre exiguë pour un loyer mensuel de 130 dollars dans un immeuble situé au milieu du camp ; le bâtiment appartient à un Libanais qui a regagné son village après le retrait israélien du sud du pays en 2000. Leur logement, dépourvu de tout équipement, était sombre et humide. Ils devaient également partager la cuisine et les sanitaires avec les autres locataires de l'étage, des Éthiopiennes qui vivaient en colocation. Trois couples soudanais-éthiopiens et des colocataires éthiopiennes occupaient ainsi les trois étages de cet immeuble vétuste. Le propriétaire ne vivant pas dans le camp, les résidents devaient traiter avec un agent intermédiaire (wakil) pour le versement des loyers. Suite à un conflit de voisinage entre les Éthiopiennes de l'immeuble

16 Situé au sud de Beyrouth, Cola constitue un hub à partir duquel il est possible de gagner les quartiers ouest et le centre de Beyrouth, la banlieue orientale et méridionale, ainsi que le nord et le sud du pays. 
dans lequel T. était impliqué, le wakil a donné un mois au couple pour quitter leur logement. Une situation qui les a mis dans l'embarras du fait de l'absence de logement vacant et dans un contexte où tout écart aux règles - notamment la discrétion - peut aboutir à l'expulsion du camp. Interrogé à ce sujet, M., nous dit :

"Tu sais les gens ici ne font pas de problèmes. C'est un camp tranquille. [...] C'est simple, l'objectif des habitants de ce camp est d'être les plus discrets possible. Alors quand les étrangers causent des problèmes, ils doivent tout faire pour les arrêter. Ils ne veulent pas déranger, ils ne veulent donc pas qu'on les dérange. Je comprends cette situation. Ce n'est pas facile pour eux. Moi je suis pareil. Quand il y a des problèmes, je cherche à les éviter. Quand les gens te respectent, tu dois les respecter. [...] Ici tout le monde me connaît par mon nom et je salue tout le monde. Je suis connu dans le camp. Je n'ai de problème avec personne " (Camp de Mar Elias, juin 2012).

Selon M., c'est grâce à sa bonne réputation au sein du camp que le couple parvient à retrouver in extremis un appartement exigu à l'entrée ouest de Mar Elias pour un loyer de 300 dollars US (220 euros). Un emplacement qui convient à $M$. qui, même s'il dit apprécier la vie dans le camp et accepter les règles et les contraintes liées à la vie en promiscuité, considère malgré tout le contrôle social au quotidien comme pesant. Résider en bordure du camp permettrait ainsi d'éviter la traversée des ruelles et de fuir les regards inquisiteurs auxquels s'expose tout résident. Le discours de M., à propos de la vie dans le camp n'est donc pas sans contradictions ni ambiguïtés. Et si certains migrants expriment clairement leur malaise face à un contrôle qu'ils jugent bien trop strict, d'autres le considèrent comme un gage certain de sécurité.

J., une jeune malgache arrivée au Liban en 2007 et en situation irrégulière depuis 2010, s'est installée en septembre 2012 à Mar Elias. Femme de chambre dans un hôtel du quartier de Verdun et sans logement fixe depuis plusieurs mois, elle trouve, grâce à un de ses collègues soudanais qui vit à Mar Elias une chambre d'environ six mètres carré, pour un loyer de 200 dollars US (145 euros) ; un prix qu'elle juge convenable au regard de ceux pratiqués à Beyrouth. J. s'installe donc chez S., un Palestinien qui a construit un bâtiment de deux étages destinés à la location pour les migrants. Aucun acompte n'est exigé par le propriétaire qui n'est pas regardant sur la situation administrative de J., ce qui la met en confiance. En effet, lors de l'entretien, J. évoque la peur qu'elle a des autorités du fait de sa situation et la crainte des agressions dans une ville où elle lit menace et racisme dans le regard porté sur les migrantes dans les espaces publics. Elle nous dit apprécier les règles strictes du camp et l'attitude paternaliste de S. qui la rassurent :

" [...] Quand j'ai décidé de venir habiter ici, un collègue libanais m'a dit "mais tu es folle, pourquoi tu vas dans un camp? Ils sont fous là-bas ! Ils boivent et font des problèmes, surtout aux femmes". Comme je ne connais rien à la Palestine, j'ai demandé conseil à ma belle-mère qui travaille depuis longtemps à Beyrouth, mais elle ne savait pas non plus. Puis quand on m'a dit Mar Elias, j'ai compris que c'était chrétien et ça m'a déjà rassurée ${ }^{17}$. [...] Je ne connais pas encore très bien le camp, mais une fois dedans je me suis sentie en sécurité. Les rues sont propres et calmes. Personne ne te dit rien. [...]

Regarde, de toute façon il y a trois portes à ouvrir pour arriver chez moi. Je dors donc tranquillement " (Camp de Mar Elias, septembre 2012).

17 Mar Elias signifie Saint Élie. 
Ces différentes perceptions, parfois ambivalentes, de la vie dans le camp reviennent fréquemment dans les entretiens. D'une part les migrants mettent en avant un respect mutuel, une condition partagée avec les Palestiniens; ils vont jusqu'à se revendiquer d'une même " communauté de statut " (Jaber, $2005: 21$ ), celle des étrangers pauvres et sans droits. Mais d'autre part, la relation avec ces hôtes très regardants sur le mode de vie au sein du camp implique une hiérarchisation très présente qui n'est pas sans rappeler celle qui prévaut dans le reste de la ville. Les travailleurs-migrants sont considérés comme de nouveaux venus sans aucun pouvoir, mais également sans aucune étiquette politique. Autant de critères qui participent à faire de ces locataires des personnes privilégiées et recherchées par des propriétaires en quête de rente immobilière.

\section{Discrimination et rente immobilière en temps de crise}

Les camps palestiniens, bien que construits par et pour les réfugiés palestiniens en territoire libanais, ont très tôt accueilli des Libanais, des Syriens, des Égyptiens et des ressortissants du monde indien (Sayigh, 1994 : 100). Cette mixité nationale et confessionnelle ancienne n'empêche pas le maintien de distinctions selon une grammaire des légitimités héritée en partie des guerres libanaises (Abou-Zaki, 2008). On retrouve aujourd'hui dans les discours des propriétaires et des responsables palestiniens interrogés, notamment à Mar Elias, cette hiérarchisation fréquemment opérée d'une part entre les résidents arabes, et d'autre part entre ces derniers et les travailleurs-migrants. En effet, quand le président du Comité populaire de Mar Elias évalue la population du camp, il l'estime à environ 1500 habitants, confondant Palestiniens et Libanais. Les Syriens, bien que présentés comme "frères ", sont de facto comptabilisés à part. Et si les autorités affichent une volonté de privilégier les ressortissants arabes et au premier chef les Palestiniens, les stratégies locatives des propriétaires paraissent en revanche avantager les migrants africains et asiatiques. Une tendance difficile à contrôler pour les autorités palestiniennes qui n'ont que peu de pouvoir sur les propriétaires, d'autant plus s'ils sont libanais. Cette volonté serait d'ailleurs aussi à nuancer alors que certains membres du Comité populaire ont adopté ces stratégies.

Ainsi S. (voir supra), ancien cadre de I'UNRWA, a décidé à sa retraite de rénover son logement et d'agrandir le bâtiment initial. Il a pu loger son frère et sa mère dans deux appartements distincts, et construire deux étages supplémentaires constitués de vingt chambres d'environ six mètres carré, et des sanitaires et cuisines communs à chaque niveau. Cette nouvelle construction, comme tous les chantiers récents d'extension du bâti dans le camp, est illégale ${ }^{18}$. Beit al-Abyade (la maison blanche), comme S. se plaît à nommer ce qui est devenu un immeuble imposant, s'achève en mars 2012. Chaque chambre est louée 200 dollars US uniquement à des ressortissants africains et asiatiques. Pour S., cette discrimination à l'encontre des Arabes - Palestiniens comme Libanais ou Syriens - est tout à fait justifiée dans le contexte social, économique et politique qui prévaut :

18 Les Palestiniens n'ont en principe pas le droit de construire sans la permission des autorités libanaises. Mais selon S., les habitants ne demandent généralement aucune autorisation, qu'il est par ailleurs difficile sinon impossible d'obtenir, ou alors l'acquièrent par la corruption des agents de police qui ne peuvent que constater les travaux déjà entamés. En outre, l'évêché propriétaire du terrain ne s'implique plus dans la gestion du camp (Sfeir, 2008 : 241). 
"C'est vrai que je ne veux pas d'Arabes. Les Arabes vont te dire qu'ils n'ont pas de travail ou que telle personne paiera à leur place. Comment je fais moi ? ! Je dois rembourser l'emprunt qui m'a permis de construire, aider ma famille. Je ne peux pas accepter que les gens ne paient pas. On ne peut pas appeler la gendarmerie pour les sortir.

Et si tu essaies, tu dois embrasser 500 mains pour négocier. Comme il n'y a pas d'État au Liban, je ne peux pas porter plainte. Si le locataire est membre de Fatah, tu vas voir l'organisation, ils te disent: "le pauvre, trouve-lui une autre maison si tu l'expulses. Où va-t-il aller sinon ?". Mais je ne suis pas Caritas ! Et puis les Palestiniens veulent plus d'électricité, plus d'eau, plus de place. Les migrants, eux, rentrent le soir, mangent et dorment. Pendant douze jours sans électricité en juillet, ils n'ont rien dit. Les Arabes, tu loues à un, il te ramène sa famille et ses amis. C'est simple, je préfère louer deux mois à un migrant que douze mois à un Arabe. C'est un casse-tête, car à Mar Elias, tu dois être à la fois la loi, la police, le propriétaire et le juge pour t'en sortir [...]" (Mar Elias, septembre 2012).

L'autre critère en faveur des migrants est le montant des loyers et la facilité de les expulser sans préavis. Les propriétaires, alors en position de choisir leurs locataires, demandent systématiquement des montants supérieurs à ce qu'ils pratiqueraient pour des Arabes. Les colocations constituent aussi un avantage pour les propriétaires qui exigent alors des sommes supplémentaires. La présence de femmes seules n'est pas un problème, mais elles doivent obéir à des règles strictes sous peine de se voir expulser : ne jamais recevoir d'homme dans leur logement sauf si les femmes sont mariées, circuler dans le camp en tenue " décente ", ne pas rentrer tard le soir, ne pas faire de bruit ${ }^{19}$.

Si la stratégie immobilière de $\mathrm{S}$. est originale à Mar Elias puisqu'elle a été planifiée spécifiquement pour louer des logements à des travailleurs non arabes, Abu M., un autre propriétaire qui loue également à des migrants, souligne quant à lui que la location à des étrangers est généralement la conséquence de la crise économique qui touche les Palestiniens et le départ des résidents du camp, principalement les jeunes qui n'ont aucun débouché professionnel au Liban. Abu M. s'est installé à Mar Elias en 1995 après l'expulsion des Palestiniens du Koweït en 1991 puis de Libye. Maintenant bien établi au sein du camp, il a pu envoyer trois de ses cinq enfants en Europe, qui à l'instar de bien d'autres jeunes réfugiés tentent ainsi un nouvel exode (Puig, 2012). Les deux étages supplémentaires initialement construits pour la famille se trouvent donc vacants. Une dizaine de locataires éthiopiennes occupent les différentes pièces du bâtiment, Abu M. refusant la mixité homme-femme et la présence d'Arabes pour les mêmes raisons que celles évoquées par $\mathrm{S}$.

S. et Abu M., tous deux rentiers et résidents, sont des " propriétaires occupants et bailleurs " selon la formule de Durand-Lasserve (1986: 41). À l'image du propriétaire libanais absent qui loue l'intégralité de son immeuble via un intermédiaire (voir supra), il existe aussi à Mar Elias la catégorie du " propriétaire bailleur et investisseur " (ibid.).

Une des conséquences de la demande croissante de logement de la part de personnes extérieures au camp - travailleurs migrants et réfugiés syriens -, est

19 Déroger à ces règles de bonnes mœurs peut avoir des conséquences importantes. Ainsi en 2005, les migrants non arabes ont été expulsés afin de couper court à une rumeur de prostitution qui entachait la réputation de Mar Elias. 
que les loyers connaissent une augmentation importante. Mais une autre conséquence de l'installation de migrants réside dans la transformation de Mar Elias et cette tendance met aussi en évidence les contradictions inhérentes à la réalité contemporaine des camps et de leurs habitants, comme le souligne le président du Comité populaire :

"Nous avons demandé aux propriétaires de louer les habitations à des Palestiniens, des Libanais ou des Syriens, mais ils nous répondent toujours que les Arabes ne paient pas à temps, ou pas du tout. [...] Nous avons une attitude de générosité et de soutien vis-à-vis des migrants. Nous sommes des étrangers, ils le sont aussi. Et puis surtout, le

Comité ne peut pas empêcher la venue des migrants dans le camp. II doit soutenir les habitants, pas les empêcher de vivre. Depuis 2005 et la mort du Martyr Rafic Hariri20, les Palestiniens ont de gros problèmes économiques et les familles n'ont que peu de choix pour s'en sortir. Les Palestiniens ne peuvent pas travailler comme les Libanais. [...] II y a des contradictions évidentes entre les intérêts individuels et collectifs dans les camps qui sont et doivent rester des lieux symboles de la résistance et de l'identité des réfugiés palestiniens " (Mar Elias, mai 2012).

Le responsable du Comité tente d'une part de justifier la préférence des autorités pour les populations arabes, et d'autre part les conditions de vie imposées et les loyers demandés aux migrants dans le camp en invoquant malgré tout l'attitude "généreuse " des résidents du fait d'une certaine altérité partagée. Finalement, deux dimensions par certains aspects contradictoires transparaissent dans ce discours. D'un côté, les réalités quotidiennes et les besoins de la population palestinienne qui réside dans la ville, mais qui ne peut prétendre aux mêmes droits que les Libanais. "Apolitiques ", car extérieurs aux enjeux régionaux et de fait sans pouvoir, les migrants deviennent ainsi une source de rente pour les propriétaires du camp qui tentent donc de pallier les difficultés économiques du quotidien. De l'autre côté, c'est l'idée même de l'espace comme symbole, l'identité du lieu et de la population réfugiée qui se voit ainsi interrogée dans le temps long de l'attente. Cette identité palestinienne dans l'exil, dont les comités populaires doivent malgré tout être les garants, se trouve comme en opposition avec la réalité irrépressible de l'argent nécessaire à la vie des habitants dans la ville. Cela se matérialise ici par la mise en place d'un système économique rentier basé sur le développement de stratégies immobilières au sein même des camps. Mais cette situation de mise en tension et de reformulation des enjeux socio-spatiaux est tout à fait comparable à Sabra où, dans un cadre résidentiel et marchand, on assiste également à une transformation des paysages urbains inhérente à l'arrivée de nouveaux acteurs dans des espaces pauvres et sous tension.

\section{Place marchande et marchandage des places}

Le marché bangladais de Sabra est un exemple de ces nouvelles sociabilités et appropriations qui contribuent " à faire " la ville dans ses marges spatiales et temporelles. Les migrants profitent de cet espace marchand informel pour se faire une place à la fois comme commerçants et clients. Toutefois, leur présence reste sous le contrôle des responsables locaux patentés qui gèrent le marché. Ils

20 Premier ministre libanais assassiné en février 2005. 
sont susceptibles d'offrir des opportunités d'installation comme de restreindre l'accès à un espace déjà très densément occupé. Le statut des migrants, sans droit, accentue leur vulnérabilité au sein d'une hiérarchie sociale et politique où les Palestiniens et les Libanais représentent les figures dominantes. Mais c'est également cette représentation " apolitique " des migrants et l'argent qu'ils versent pour se voir attribuer un emplacement qui leur permettent de s'installer plus ou moins durablement sur un marché tel que Sabra.

\section{Le marché bangladais de Sabra}

Qui se rend à Sabra le dimanche matin ne peut être que surpris par l'ambiance du marché. En ce jour chômé pour les travailleurs migrants, les commerçants bangladais invitent le chaland à venir acheter des cd de musique et de films, des légumes et des fruits exotiques importés ou cultivés au Liban essentiellement dans la région de Choueifate (sud de Beyrouth) -, des poissons, des épices et autres produits indiens. Mais trouver et conserver une place sur le marché, où sont déjà rassemblés et organisés les taxis, les bouchers, les vendeurs ambulants syriens, palestiniens et libanais, s'avèrent compliqué pour ces nouveaux venus. II est d'ailleurs fréquent d'assister à d'âpres négociations entre ces différents acteurs pour l'obtention d'un peu d'espace-temps, certes jamais définitif, mais toujours dûment monnayé. Autant d'interactions et de transactions qu'il est difficile d'analyser pour un observateur extérieur lors d'une territorialisation si brève et si intense, et du fait de l'informalité des règles et de l'ambiguïté des discours.

Parmi les vendeurs bangladais, deux catégories peuvent être distinguées: les vendeurs du dimanche et les commerçants. Ceux que je nomme " vendeurs du dimanche " sont les plus visibles. Ils sont une vingtaine d'hommes, à l'exception d'un couple ${ }^{21}$, qui sont présents uniquement le dimanche matin ; ils sont employés la semaine dans des sociétés d'entretien, des stations essence ou des industries. Équipés de cagettes, de cartons et de tables en plastique pour exposer leur marchandise, ils tentent de conserver leur place sur le marché où ils espèrent compléter leurs revenus (voir Photo 1). Certains ont un emplacement en principe réservé, sur un coin de trottoir, rétribuant les commerçants libanais et palestiniens devant lesquels ils s'installent. Ils doivent également s'acquitter d'une somme auprès des "placeurs " du marché, présentés par les vendeurs comme des " mafieux " chargés de taxer les commerçants pour le compte des autorités qui contrôlent le quartier. Les derniers arrivés n'ont de place qu'au milieu du carrefour, au bord de la route, coincés entre les allers et venues des bouchers - majoritaires dans ce secteur -, des automobilistes et des scooters. Ils se déplacent régulièrement pour éviter de voir leur marchandise abîmée ou pour laisser la place aux taxis et aux commerçants palestiniens et libanais. Ces vendeurs tentent cependant de conserver leur emplacement, subissant les invectives, parfois violentes, des vendeurs ambulants arabes - palestiniens, libanais, syriens - anciennement installés : "Pousse-toi Bangladais, tu vois bien

\footnotetext{
21 Selon des vendeurs interrogés, cette situation n'est que la transposition de la pratique de la purdah, autrement dit un accès inégal entre hommes et femmes à l'espace public, ces dernières devant rester à l'abri des regards étrangers. Par conséquent, les femmes sont généralement peu présentes dans les espaces marchands au Bangladesh, en tant que commerçantes, mais aussi en tant que clientes. Ce qui expliquerait l'inégale représentation des sexes sur le marché de Sabra.
} 
que tu empiètes sur ma place ! ". Ce propos fréquemment entendu sur le marché de Sabra tend à réaffirmer les formes de hiérarchie tant ethnique que sociale qui à la fois caractérise et organise le marché. Les vendeurs à l'emplacement plus stable sont également susceptibles de subir la rudesse des commerçants qu'ils ont pourtant rémunérés ; les étals des Bangladais et leurs clients obstruent régulièrement l'accès aux magasins. Une tension est donc toujours palpable. Elle se répercute ponctuellement sur les rapports entre les migrants dans un contexte de promiscuité, de concurrence et comme conséquence de la pression des " placeurs " qui menacent de les expulser s'ils refusent de payer et de les remplacer par d'autres Bangladais.

\section{Photo 1 : Commerçants bangladais sur le marché de Sabra}

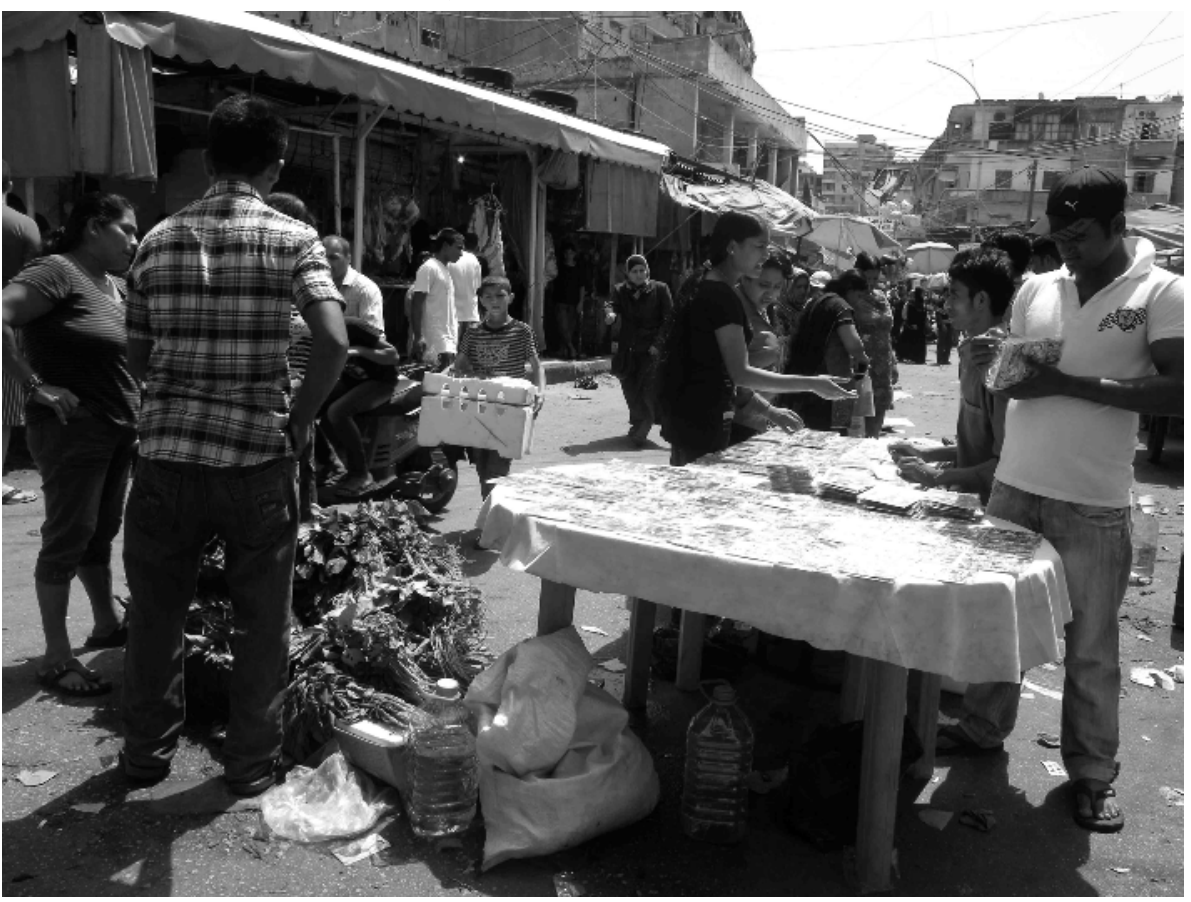

Crédit : Assaf Dahdah, 2013.

L'installation des "vendeurs du dimanche " résulte d'une transformation plus ancienne initiée par Sa., un ouvrier d'une quarantaine d'années arrivé du Bangladesh en 1993 et qui dès 2003 complétait son salaire de nettoyeur de frigos industriels par la vente de produits du monde indien à l'entrée du groupement Da'ouk. Depuis 2010, Sa. assiste à l'arrivée de centaines de ses compatriotes dans le secteur de Sabra et Chatila, et à l'augmentation du nombre de vendeurs sur le marché où le commerçant fait figure de pionnier. Sa petite échoppe, louée 200 dollars US (145 euros), est constituée d'une petite armoire et d'un étal de fruits et légumes. À la faveur de l'ancienneté, le commerçant bénéficie d'une reconnaissance auprès des habitants de $\mathrm{Da}^{\prime}$ ouk et des commerçants voisins, certains le gratifiant de titres honorifiques tel que " représentant des Bangladais de Sabra " (mukhtar al-bangladeshiyine bi Sabra). Sa. tient à sa discrétion qu'il 
estime être la garantie de sa place et de sa longévité dans un espace disputé. Une discrétion qu'il dépeint malgré tout comme le résultat d'une soumission, même s'il évoque la présence des Bangladais à Sabra comme une cohabitation relativement " horizontale " entre des étrangers relégués et pauvres :

"Avant, c'était un Syrien qui vendait des parfums dans cette armoire. Mais il est parti alors j'ai pris sa place en 2003. C'est vrai que c'est cher pour ce que c'est, mais je suis tranquille. Quand la municipalité vient pour prélever les taxes des commerçants, ou la police pour dégager les vendeurs de rue, moi je n'ai pas de problèmes. Les autres doivent tout ranger et disparaitre le temps de la descente. Moi, je suis dans mon coin, personne ne fait attention à moi. [...] Ici les Bangladais sont bien. II n'y a pas de racisme, nous n'avons pas de problème, car je pense que tout le monde est au même niveau. Les Palestiniens sont pauvres, nous aussi. Et puis nous sommes discrets, on ne veut pas faire de problèmes. Si on nous bouscule, on s'excuse et on fait ce que l'on nous demande "

(Sabra, mai 2012).

Resté très évasif à ce sujet, Sa. doit malgré tout se soumettre à la " protection " des responsables locaux en leur reversant régulièrement une taxe pour conserver son commerce ouvert tous les jours de la semaine. II doit aussi affronter la concurrence des nouveaux commerçants présents sur le marché. Car comme lui, et en plus des vendeurs du dimanche, quatre autres commerces bangladais ont aujourd'hui pignon sur rue à Sabra, dont trois se sont installés durant la seule année 2012. Parmi eux, deux Bangladais mariés à des Libanaises louent un local commercial enregistré au nom de leur épouse et ne dépendent donc pas directement des placeurs du marché. Tous vendent quasiment les mêmes produits, Sa. étant toutefois le seul à avoir également un restaurant situé dans le groupement Da'ouk22 (voir Photo 2).

Le rassemblement bangladais à Sabra attire un nombre toujours plus important de migrants qui s'approprient cet espace tous les dimanches. Son ampleur, même s'il reste spatialement concentré, surprend encore des usagers du marché. Si certains se disent encore étonnés par la présence de ces nouveaux venus, des clients arabes ont également pris l'habitude de leur acheter des piments et des épices. Dans ce contexte, le rôle des commerçants et des responsables locaux se révèle ambigu, alliant relation lucrative, protection et menace. Les responsables tiennent à affirmer leur contrôle sur le marché et sur le quartier, rappelant fréquemment leur pouvoir de protéger les Bangladais comme celui de les expulser.

La position officielle du Comité populaire de Da'ouk consiste donc à refuser aux propriétaires la location immobilière à des migrants dans le groupement. Un principe dicté par la volonté affichée du Comité de " préserver " l'identité palestinienne sinon arabe des lieux, dont l'ouverture du restaurant bangladais de Sa. constitue cependant un contre-exemple. À l'image de Mar Elias et du marché, les réalités économiques et sociales incitent au compromis, et la présence des Bangladais au sein des groupements n'est alors présentée que comme une menace somme toute très relative et par ailleurs très lucrative.

22 Lors du dernier terrain mené en janvier 2014, Sa. avait toutefois vendu son restaurant à un Bangladais pour la somme de 4000 dollars US. 
Photo 2 : Le restaurant bangladais dans le groupement Da'ouk

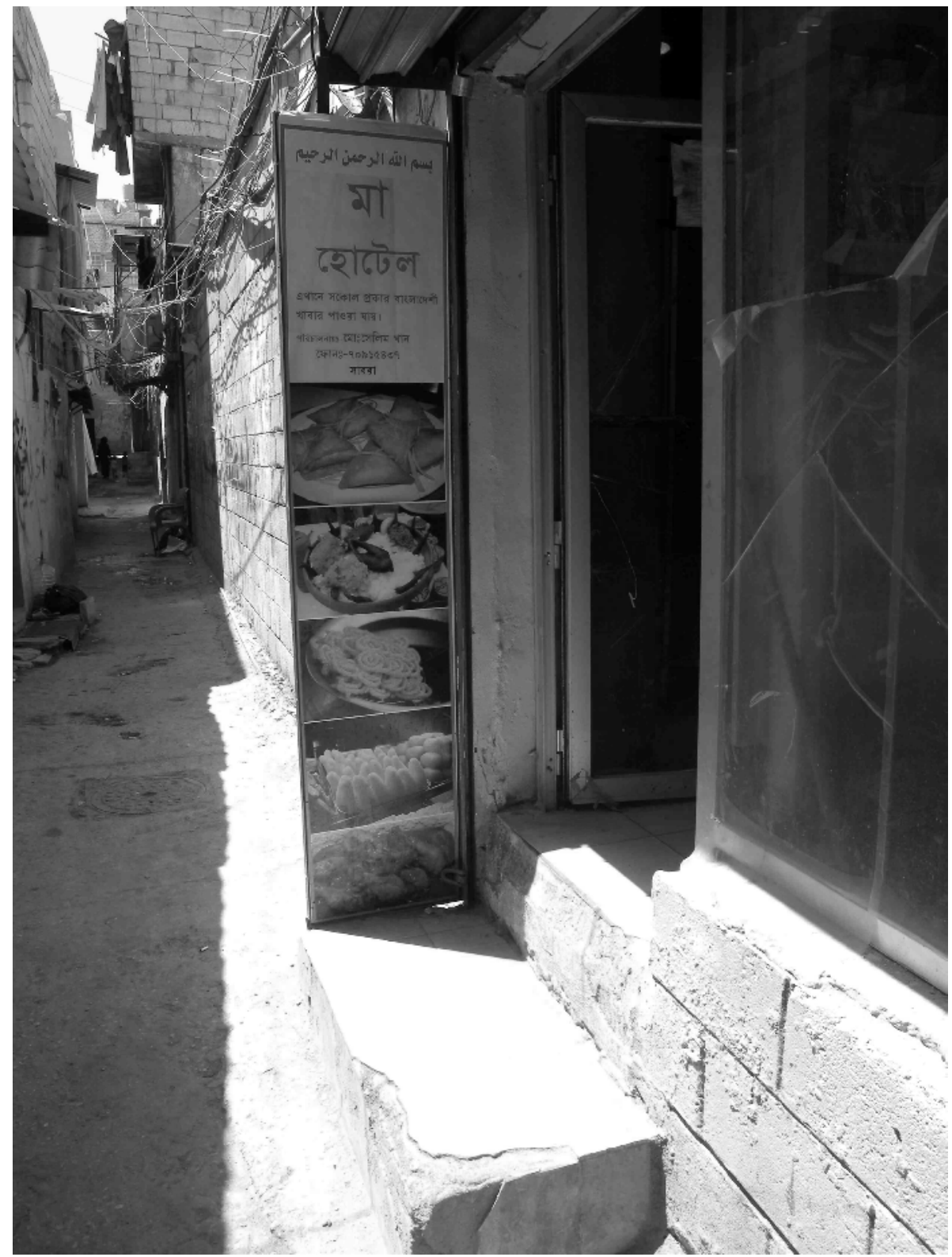

Crédit : Assaf Dahdah, 2013. 


\section{À chacun sa place : négocier la présence de l'autre dans des espaces sous tension}

"Ce n'est plus Sabra ici, c'est Dacca! " Par cette remarque, un commerçant de Sabra, faisant mine d'être exaspéré par la présence importante des Bangladais le dimanche matin, met en exergue les transformations socio-spatiales que connaît le quartier. Cependant, l'installation des travailleurs migrants ne représente pas un enjeu local majeur pour les Palestiniens et les Libanais de Sabra qui assistent et pour certains participent à leur insertion sur le marché et dans les quartiers avoisinants. Mais c'est avant tout l'arrivée massive et concomitante de réfugiés syriens et de réfugiés palestiniens de Syrie qui alimente la crainte des habitants de Sabra de se voir " envahir " durablement par tous ces " nouveaux invités ". Avec un discours officiel de solidarité, ces réfugiés ne sont jamais évoqués comme un problème. Mais dans les faits et dans un contexte de suspicion, l'afflux de milliers de personnes, notamment depuis la Syrie, bouleverse ces espaces déjà densément peuplés et paupérisés où la mixité confessionnelle et nationale peut être aussi synonyme de tensions, voire de conflit armé.

Comme je l'ai déjà évoqué à propos de Mar Elias (voir supra), la conséquence directe de cet afflux de réfugiés est l'inflation des prix des loyers, certains propriétaires complètent leurs bénéfices grâce aux aides financières distribuées par les organisations et institutions internationales aux déplacés pour se loger. Dans le cas de Sabra, on assiste également à une demande venant de ces nouveaux résidents pour intégrer même temporairement le marché comme vendeur ou employé pour gagner un peu d'argent. Les personnes recrutées sont alors très faiblement rémunérées du fait de la concurrence et les nouveaux vendeurs sont largement taxés par les " placeurs ". Enfin, la crainte de voir ces populations déplacées s'installer reste grande. Dans ce contexte, les Bangladais paraissent totalement inoffensifs, car extérieurs à tous ces enjeux régionaux et locaux, et leur appartenance à l'islam sunnite, fréquemment mentionnée par les personnes interrogées, deviendrait alors un facteur supplémentaire d'intégration dans ces quartiers. On peut d'ailleurs lire en arabe sur le panneau à l'entrée du restaurant de Sa. (voir Photo 2) la mention islamique " bism allah al-rahman al-rahim " (au nom de Dieu le Compatissant le Miséricordieux). II n'en demeure pas moins que leur appropriation de l'espace public doit être limitée et seule une activité marchande à destination des Bangladais est autorisée. Un entretien avec un boucher libanais concernant la place des " étrangers " sur le marché est à cet égard éloquent :

"[Les Bangladais] sont discrets, ils ne font pas de problèmes. Si tu leur dis quelque chose, ils t'écoutent [...], tu n'as pas besoin de négocier. Je te parle surtout des Syriens. II leur manque une case ! Dès qu'ils voient une femme, [...] quand il y a beaucoup de monde, ils essaient de la toucher, [...] alors on les frappe. Avant, quand les Syriens contrôlaient le pays, on ne pouvait pas faire ça, mais maintenant, on fait ce que l'on veut ici. [...] On ne sait jamais comment les choses vont changer. Avant c'étaient les Syriens, maintenant ce sont les Bangladais. Qui peut dire à quoi ressemblera ce marché dans cinq ans ". Puis le boucher se tourne vers un commerçant bangladais assis à proximité et lui dit: "Alors $\mathrm{H}_{\text {., }}$ tu as un deuxième parasol! Tu t'agrandis encore on dirait. Tu vas bientôt nous prendre tout le carrefour ! [...] Tu vois, je les attaque un peu, il faut bien qu'ils sentent qu'ils n'ont pas le carrefour, sinon ils vont prendre toute la place. Pour l'instant on ne se fait pas concurrence, mais si un jour l'un d'eux décide d'ouvrir une boucherie, je l'égorge ! ". 
Que ce soit à l'égard des Syriens ou des Bangladais, les commerçants établis réaffirment constamment leur pouvoir dans ces lieux où la légitimité et la place de chacun paraissent constamment menacées. Si les travailleurs syriens, généralement des ouvriers célibataires déclassés, ont relativement bénéficié de I'occupation militaire et de la mise sous tutelle du Liban par le régime baasiste (Blanc, 2006), cette période est maintenant révolue ${ }^{23}$. Or, comme le souligne notre boucher, la hiérarchie momentanément bouleversée est désormais réinstaurée, mais aucune certitude quant à la pérennité de cette remise en ordre n'est permise sur un marché tel que Sabra. Même présenté dans un premier temps comme inoffensif et utilisant malgré tout le ton de l'humour, le message qu'il envoie à l'intention du commerçant bangladais reste clair : à chacun sa place.

Dans un contexte de paupérisation et de concurrence spatiale, analyser l'insertion de nouveaux acteurs dans un espace commercial tel que Sabra met en lumière des sociabilités complexes, mouvantes et des rapports de force parfois extrêmement violents. À l'image de ce nouveau marché bangladais, I'informalité, les inégalités, la précarité et les incertitudes politiques qui animent les quartiers pauvres constituent donc des vecteurs d'opportunités et d'insertion pour tout nouvel arrivant. Mais elles sont également des sources d'instabilité et de perpétuation de l'exclusion aux marges spatiales et sociales de la ville.

\section{Conclusion}

Étudier la place des migrants dans les espaces palestiniens implique deux analyses concomitantes : celle des trajectoires sociales et spatiales incertaines des travailleurs non arabes à Beyrouth, et la transformation de ces espaces au sein de la métropole. Conséquence des modalités d'entrée sur le marché du travail libanais, la place des migrants dans la ville est marquée par un déclassement social et des discriminations ethniques. Leurs capacités à s'insérer dépendent donc des opportunités individuelles et collectives qu'ils parviennent à développer dans les interstices de la ville. Néanmoins, en tant que personnes sans droit, cette volonté ne peut qu'être soumise à celle des acteurs dominants qui contrôlent les espaces où ces stratégies sont susceptibles de se déployer. Les marges géographiques de Beyrouth sont privilégiées parce qu'à l'écart et paupérisées. Ces quartiers informels offrent alors des places pour quiconque est en mesure d'en accepter le fonctionnement régi par des négociations permanentes entre acteurs inégaux.

Les migrants, sujets de stigmatisations récurrentes et multiples, acquièrent dans les camps palestiniens un statut particulier, celui de l'étranger sans pouvoir, mais aussi extérieur aux enjeux politiques locaux, nationaux et régionaux. Tout en étant hors et dans la ville, camps et groupements deviennent ainsi des destinations privilégiées pour les migrants qui y trouvent des conditions d'installation appropriées. Mais leur place n'y est jamais assurée. Les discours des acteurs dominants - autorités locales, propriétaires, commerçants et " placeurs " - révèlent dès lors l'instabilité et la faiblesse de leur position, de même que l'incertitude de leur présence sous caution. Car in fine, dans ces quartiers pauvres, la garantie effective de la présence des travailleurs migrants

23 Pour plus de détails, lire également l'article de Balanche (2007). 
réside en grande partie dans les intérêts économiques de certains habitants. Espaces d'insertion et d'opportunités, les camps et les groupements palestiniens et le marché de Sabra n'en restent pas moins des espaces de fixation des statuts entre populations déclassées. Des réalités perturbant l'identité même de ces lieux qui deviennent des quartiers urbains, pauvres, parmi d'autres. Et où l'idée noble de la résistance politique et le rêve du retour se substituent à l'évidence flagrante d'une dilution progressive dans la banalité de la ville, de la marginalisation et de l'exploitation capitaliste.

Appréhender la place des migrants dans les marges de Beyrouth implique donc de déplacer le regard porté habituellement sur la ville et de se concentrer sur le rôle des habitants en tant qu'acteurs et producteurs d'une urbanité. Étudier leur présence dans les camps et groupements palestiniens signifie s'intéresser aux " contre-espaces " (Cattan et Clerval, $2011: 26$, citant Chetcuti, 2010), ces lieux en marge des quartiers lumineux censés être la synecdoque de la ville (Santos, 1997). Comme je l'ai montré, la production de la ville ne se limite pas aux centralités formelles, mais elle s'invente aussi dans les marges de Beyrouth, quartiers pauvres dont les travailleurs migrants constituent de nouveaux acteurs. Ils participent à une " urbanité des marges " qui s'élabore notamment dans les espaces palestiniens (Puig et Doraï, 2012). Une urbanité qui reste caractérisée par la hiérarchisation, la précarité des statuts et les stigmatisations ethniques, par des "informalités et des inégalités cumulatives [...] [qui renforcent] les processus d'exclusion, eux-mêmes générateurs et révélateurs de profondes inégalités sociales, économiques, citoyennes " (Baby-Collin, 2010 : 9).

\title{
Références bibliographiques
}

\begin{abstract}
Abou-Zaki Hala Caroline (2008) Les Palestiniens du Liban après Taëf. Transformations sociopolitiques dans le camp de Chatila au lendemain de la guerre des camps (1985-1987) et de la guerre interne (1988), Mémoire de Master, Paris, EHESS, $194 \mathrm{p}$.
\end{abstract}

Agier Michel (2013) Campement urbain. Du refuge naît le ghetto, Paris, Payot \& Rivages, $133 \mathrm{p}$.

Agier Michel (2012) Temps, espace et politique. Éléments d'ạnthropologie des camps de réfugiés, in Mohamed Kamel Doraí et Nicolas Puig Éds, L'urbanité des marges. Migrants et réfugiés dans les villes du Proche-Orient, Paris/Beyrouth, Éditions Ifpo/Téraèdre, pp. 29-38.

Al Husseini Jalal (2012) Les camps de réfugiés palestiniens, soixante ans après : évolution des discours, adaptations des pratiques, in Mohamed Kamel Doraï et Nicolas Puig Éds, L'urbanité deș marges. Migrants et réfugiés dans les villes du Proche-Orient, Paris/Beyrouth, Éditions Ifpo/Téraèdre, pp. 39-62.

Al Husseini Jalal et Doraï Kamel (2013) La vulnérabilité des réfugiés palestiniens à la lumière de la crise syrienne, Confluences Méditerranée, 87 (4), pp. 95-107.

Baby-Collin Virginie (2010) Inégalités et informalités dans les Amériques, in Les Papiers de l'axe Territoires, Villes, Modes de vie, Environnement, 6, Toulouse, MSH Toulouse/ATRIA, pp. 9-24.

Blanc Pierre (2006) De la tutelle à la coopération ? La difficile transition des relations syro-libanaises, Confluences Méditerranée, 56 (1), pp. 55-70. 
Bonnemaison Joël (2000) La géographie culturelle, Paris, Éditions du CTHS, $152 \mathrm{p}$.

Bourgey André et Pharés Joseph (1973) Les bidonvilles de I'agglomération de Beyrouth, Revue de géographie de Lyon, 48, pp. 107-139.

Bret Julien (2011) Travail global et production d'un individu servile. Activités économiques et migrations de travailleurs non arabes au Liban, Thèse de doctorat en sociologie, Lyon, Université Lumière Lyon 2, 440 p.

Cattan Nadine et Clerval Anne (2011) Un droit à la ville ? Réseaux virtuels et centralités éphémères des lesbiennes à Paris, justice spatiale / spatial justice, 3, pp. 17-45.

Chalcraft John (2009) The invisible cage. Syrian migrant workers in Lebanon, Stanford, Stanford University Press, $310 \mathrm{p}$.

Chetcuti Natacha (2010) Se dire lesbienne. Vie de couple, sexualité, représentation de soi, Paris, Payot \& Rivages, 304 p.

Cox Rosie (2006) The Servant Problem. Domestic Employment in a Global Economy, New York-London, I.B. Tauris, 163 p.

Dahdah Assaf (2014) "Maid in Lebanon". Lebanon and the world domestic service market, in Lisa Anteby, Virginie Baby-Collin, Sylvie Mazzella, Stéphane Mourlane, Cédric Parizot and Pierre Sintès Eds., Borders, Migrations and mobilities. Perspectives from the Mediterranean, Berne, Peter Lang, pp. 131-148.

Deboulet Agnès (2012) Impasses résidentielles et citoyennetés en berne : migrants internationaux à Beyrouth, in Mohamed Kamel Doraï et Nicolas Puig Éds, L'urbanité des marges. Migrants et réfugiés dans les villes du Proche-Orient, Paris/Beyrouth, Éditions Ifpo/Téraèdre, pp. 151-180.

Deboulet Agnès et Hily Marie-Antoinette (2009) Les migrants de Beyrouth. Emplois à bon marché et zones refuges, EchoGéo, 8, [en ligne]. URL : http:// echogeo.revues.org/index10944.html

De Souza Araujo Dias Amanda (2009) Du moukhayyam à la favela. Une étude comparative entre un camp de réfugiés palestiniens au Liban et une favela carioca, Thèse de doctorat en sociologie, Paris, EHESS, $371 \mathrm{p}$.

Doraï Mohamed Kamel (2010) Palestinian Refugee Camps in Lebanon. Migration, Mobility and the Urbanization Process, in Are Knudsen and Sari Hanafi Eds., Palestinian Refugees. Identity, Space and Place in the Levant, London, Routeledge, pp. 67-81.

Doraï Mohamed Kamel (2006) Les réfugiés palestiniens du Liban. Une géographie de l'exil, Paris, CNRS éditions, 252 p.

Durand-Lasserve Alain (1986) L'exclusion des pauvres dans les villes du Tiersmonde, Paris, L'Harmattan, $198 \mathrm{p}$.

Fawaz Mona (2007) Apogée et déclin d'une nouvelle classe de citadins : les lotisseurs dans une banlieue irrégulière de Beyrouth, in Isabelle Berry-Chikhaoui, Agnès Deboulet et Laurence Roulleau-Berger Éds., Villes internationales. Entre tensions et réactions des habitants, Paris, La Découverte, pp. 223-242.

Gardner Andrew (2010) Engulfed: Indian Guest Workers, Bahraini Citizens, and the Structural Violence of the Kafala System, in Nicholas De Genova andt Nathalie Peutz Eds., The Deportation Regime. Sovereignty, Space, and the Freedom of Movement, Durham and London, Duke University Press, pp. 196-223. 
Geisser Vincent (2012) La terre promise... mais pas pour tout le monde. De la fiction à la réalité de la xénophobie anti-africaine en Israël, Migrations société, 141-142, pp. 3-13.

Hanafi Sari (2006) Vivre dans le camp, vivre ailleurs : Les Palestiniens réfugiés en Égypte et dans les Territoires palestiniens, Bulletin de l'association des géographes français, 83, pp. 79-92.

Hanafi Sari et Long Taylor (2012) Gouvernance, gouvernementalité et état d'exception dans les camps de réfugiés au Liban, in Mohamed Kamel Doraï et Nicolas Puig Éds, L'urbanité des marges. Migrants et réfugiés dans les villes du Proche-Orient, Paris/Beyrouth, Éditions Ifpo/Téraèdre, pp. 63-85.

Harb Mona (2003) La Dâhiye de Beyrouth : parcours d'une stigmatisation urbaine, consolidation d'un territoire politique, Génèses, 51, pp. 70-91.

Jaber Hanna (2005) Introduction, in Hanna Jaber et France Métral Éds., Mondes en mouvements. Migrants et migrations au Moyen-Orient au tournant du XXIe siècle, Beyrouth, Ifpo, pp. 10-25.

Jureidini Ray (2003) L'échec de la protection de l'État : les domestiques étrangers au Liban, Revue Européenne des Migrations Internationales, 19 (3), pp. 95-127.

Longuenesse Élisabeth (2013) Travailleurs étrangers, droit du travail et réglementation de l'immigration, Les carnets de I'lfpo, [en ligne] consulté le 18/11/2013. URL : http://ifpo.hypotheses.org/5393

Moukarbel Nayla (2009) Sri Lankan Housemaids in Lebanon: a case of "symbolic violence" and "everyday forms of resistance", Amsterdam, Amsterdam University Press, 245 p.

Navez-Bouchanine Françoise (2001) Des villes entre fragmentation spatiale et fragmentation sociale : une approche critique de la notion de fragmentation, in Élisabeth Dorier-Apprill Éd., Vocabulaire de la ville. Notions et références, Paris, Éditions du Temps, pp. 109-118.

Puig Nicolas (2012) Le voyage en Grèce. Itinéraires allers-retours de Palestiniens réfugiés au Liban, Métropolitiques, [en ligne] consulté le 05/06/2012. URL : http:// www.metropolitiques.eu/Le-voyage-en-Grece-Itineraires.html

Puig Nicolas et Doraï Mohamed Kamel (2012) Introduction. Insertions urbaines et espaces relationnels des migrants et réfugiés au Proche-Orient, in Mohamed Kamel Doraï et Nicolas Puig Ẽds, L'urbanité deș marges. Migrants et réfugiés dans les villes du Proche-Orient, Paris/Beyrouth, Éditions Ifpo/Téraèdre, pp. 11-25.

Santos Milton (1997) La nature de I'espace, Paris, L'Harmattan, 275 p.

Sayigh Rosemary (1994) Too Many Enemies: The Palestinian Experience in Lebanon, Londres, Zed Books, 384 p.

Sfeir Jihane (2008) L'Exil palestinien au Liban. Le temps des origines (1947-1952), Paris/Beyrouth, Karthala/lfpo, 340 p.

Soja Edward (2010) Seeking Spatial Justice, Minneapolis/London, University of Minnesota Press, $256 \mathrm{p}$.

Tachjian Vahé (2009) L'établissement définitif des réfugiés arméniens au Liban dans les années 1920 et 1930, in Aida Boudjikanian Éd., Les Arméniens du Liban : des Princesses et des Réfugiés du Passé à la Communauté Contemporaine, Beyrouth, Haigazian University and The Armenian Heritage Press, pp. 59-74. 


\section{Sources journalistiques et institutionnelles}

Balanche Fabrice (2007) Les travailleurs syriens au Liban ou la complémentarité de deux systèmes d'oppression, Le Monde Diplomatique, 6 p., [en ligne]. URL : http://hal.archives-ouvertes.fr/docs/00/28/39/11/PDF/Les_travailleurs_syriens_au_ Liban.pdf

Frantz Elizabeth (2014) Breaking the Isolation: Access to Information and Media Among Migrant Domestic Workers in Jordan and Lebanon, New York, Open Society Foundations, $37 \mathrm{p}$.

HRW (2010) Without Protection. How the Lebanese Justice System Fails Migrant Domestic Workers, New York, Human Rights Watch, 59 p., [en ligne] consulté le 11/12/2012. URL : http://www.hrw.org/sites/default/files/reports/lebanon0910webwcover.pdf

Jureidini Ray (2012) Lebanon's ways are sponsoring suicide, The Daily Star Newspaper - Lebanon, [en ligne] consulté le 10/04/2012. URL : http://www. dailystar.com.lb/Opinion/Commentary/2012/Apr-10/169709-lebanons-ways-aresponsoring-suicide.ashx\#ixzz1rkgcNBbF

UnHCR (2014) Syria Regional Refugee Response, [en ligne] consulté le 06/05/2014. URL : http://data.unhcr.org/syrianrefugees/regional.php

UNRWA (2014) Where we work. Lebanon, [en ligne] consulté le 06/05/2014. URL : http://www.unrwa.org/where-we-work/lebanon

Wehbe Elie (2012) Liban : Conflit social avec des travailleurs étrangers à Bickfaya, L'Orient-Le Jour, [en ligne] consulté le 21 mars 2012. URL : http://www.lorientlejour.com/category/\%C3\%80+La+Une/article/750969/Liban_\%3A_Conflit_social_ avec_des_travailleurs_etrangers_a_Bickfaya.html 


\section{Assaf Dahdah}

\section{Négocier sa place chez l'autre. Les migrants dans les espaces palestiniens à Beyrouth}

Au Liban, les migrants originaires d'Afrique et d'Asie s'inscrivent dans le cadre d'une mobilité économique contractualisée. Analyser leur place dans la capitale Beyrouth dépasse cependant le seul cadre professionnel pour interroger également leurs stratégies spatiales, plus particulièrement dans les marges de la ville où ils parviennent à s'installer. Entre opportunités et contraintes, appropriation spatiale et hiérarchie sociale, leurs trajectoires résidentielles et leur accès à des centralités marchandes informelles révèlent leur capacité à être malgré tout acteur de leur mobilité. Concomitamment, la présence de ces nouveaux locataires et commerçants révèle et participe à la transformation des espaces palestiniens concernés, secteurs précarisés en transition entre camp de réfugiés et ville. Ces processus sont abordés dans cet article à partir d'une étude empirique du camp Mar Elias et du quartier de Sabra.

\section{Negotiating a Place. Migrant Workers settle in Beirut's Palestinian Spaces}

In Lebanon, Asian and African migrants enter the country as contracted workers. Analyzing their role and function in Beirut goes beyond looking at their professional framework as we also examine their spatial patterns and strategies, mainly in the city outskirts where they are settling. Between opportunities and constraints, spatial appropriation and social hierarchy, their residential trajectories along with their access to informal business centers show that they remain actors in their mobility. In parallel, the settlement of such new tenants and business owners brings to light the transformation of the Palestinian spaces and their transition from a refugee's camp to becoming part of the city. Based on the empirical approach, this article examines the Mar Elias camp and the Sabra neighborhood as examples of these processes.

\section{.. Negociar su lugar en territorio ajeno. Inmigrantes en espacios palestinos de Beirut}

Los inmigrantes africanos y asiáticos en Líbano viajan bajo contratos laborales. Sin embargo, el análisis de su posición en la ciudad de Beirut no puede limitarse al simple marco profesional si no que debe extenderse al estudio de las estrategias espaciales de estos, en especial en las márgenes de la ciudad dónde la mayoría de estos viven. Entre oportunidades y obstáculos, apropiación espacial y jerarquía social, sus trayectorias residenciales y su capacidad de acceso a los centros del comercio informal revelan sus capacidades de convertirse plenamente en actores de su propia movilidad. Al mismo tiempo, la presencia de estos nuevos ocupantes en los espacios palestinos, ya sea como inquilinos o como comerciantes, atestigua y participa a la transformación de estos espacios de marginación que progresivamente transitan de campamentos urbanos a barrios. En este artículo se exponen los casos del campamento Mar Elias y del barrio de Sabra. 